Georgia State University

ScholarWorks @ Georgia State University

Summer 7-15-2011

\title{
Jackknife Empirical Likelihood for the Accelerated Failure Time Model with Censored Data
}

Maxime K. Bouadoumou

Georgia State University

Follow this and additional works at: https://scholarworks.gsu.edu/math_theses

Part of the Mathematics Commons

\section{Recommended Citation}

Bouadoumou, Maxime K., "Jackknife Empirical Likelihood for the Accelerated Failure Time Model with Censored Data." Thesis, Georgia State University, 2011.

doi: https://doi.org/10.57709/2102657

This Thesis is brought to you for free and open access by the Department of Mathematics and Statistics at ScholarWorks @ Georgia State University. It has been accepted for inclusion in Mathematics Theses by an authorized administrator of ScholarWorks @ Georgia State University. For more information, please contact scholarworks@gsu.edu. 


\title{
JACKKNIFE EMPIRICAL LIKELIHOOD FOR THE ACCELERATED FAILURE TIME MODEL WITH CENSORED DATA
}

by

\section{MAXIME BOUADOUMOU}

\author{
Under the Direction of Dr. Yichuan Zhao
}

\begin{abstract}
Kendall and Gehan estimating functions are used to estimate the regression parameter in accelerated failure time (AFT) model with censored observations. The accelerated failure time model is the preferred survival analysis method because it maintains a consistent association between the covariate and the survival time. The jackknife empirical likelihood method is used because it overcomes computation difficulty by circumventing the construction of the nonlinear constraint. Jackknife empirical likelihood turns the statistic of interest into a sample mean based on jackknife pseudo-values. $U$-statistic approach is used to construct the confidence intervals for the regression parameter. We conduct a simulation study to compare the Wald-type procedure, the empirical likelihood, and the jackknife empirical likelihood in terms of coverage probability and average length of confidence intervals. Jackknife empirical likelihood method has a better performance and overcomes the under-coverage problem of the Wald-type method. A real data is also used to illustrate the proposed methods.
\end{abstract}

INDEX WORDS: Confidence interval, Coverage probability, Jackknife empirical likelihood, Rightcensoring, U-statistic, Kendall's estimating equation Gehan, Logrank 


\title{
JACKKNIFE EMPIRICAL LIKELIHOOD FOR THE ACCELERATED FAILURE TIME MODEL WITH CENSORED DATA
}

by

\section{MAXIME BOUADOUMOU}

A Thesis Submitted in Partial Fulfillment of the Requirements for the Degree of

\author{
Master of Science \\ in the College of Arts and Sciences
}

Georgia State University 
Copyright by

Maxime Bouadoumou

2011 


\section{JACKKNIFE EMPIRICAL LIKELIHOOD FOR THE ACCELERATED FAILURE TIME}

MODEL WITH CENSORED DATA

by

MAXIME BOUADOUMOU

$\begin{aligned} \text { Committee Chair: } & \text { Dr.Yichuan Zhao } \\ \text { Committee: } & \text { Dr. Jun Han } \\ & \text { Dr. Yuanhui Xiao }\end{aligned}$

Electronic Version Approval:

Office of Graduate Studies College of Arts and Sciences Georgia State University August 2011 


\section{ACKNOWLEDGEMENTS}

This thesis would not have been possible without the support of all the people who lent me their supports in different ways. I would like to express my deepest gratitude and sincere appreciation to each and every of them. First of all, I would like to thank my advisor Dr. Yichuan Zhao for sharing his great wealth of knowledge in statistics, especially in survival analysis with me. His encouragement, insightful guidance and his patience with me are highly appreciated and always remembered.

Second of all, I would also like to thank my committee members Dr. Jun Han and Dr. Yuanhui Xiao for taking some valuable time out of their schedules to read my thesis and for giving me some critical suggestions in my thesis research.

I would also want to thank all the faculty members in the department of statistics for teaching me and helping me develop my statistical knowledge throughout my graduate study.

Thanks to all my classmates who helped me through these years and made it all bearable. Without the help of everyone, I would by no means be able to complete this research paper. 


\section{TABLE OF CONTENTS}

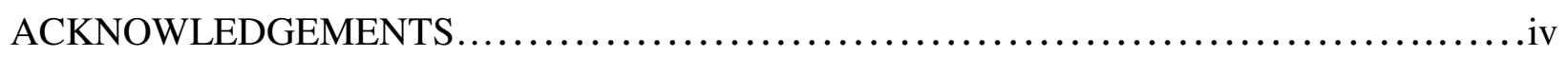

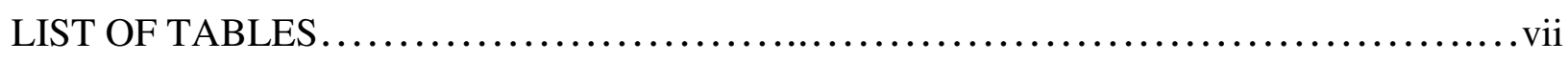
CHAPTER

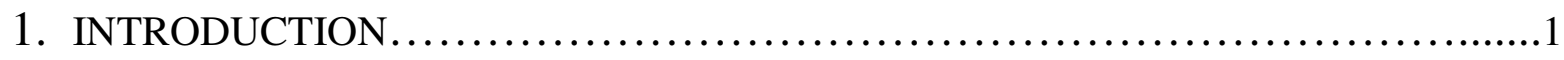

1.1 Accelerated Failure Time Model....................................................

1.2 Monotone Gehan estimating equation...........................................2

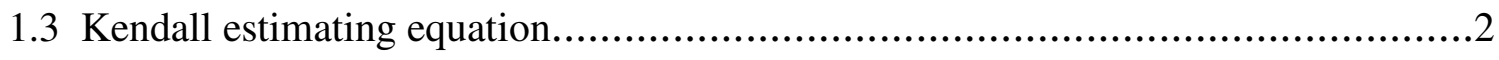

1.4 Empirical Likelihood.................................................2

1.5 Jackknife Empirical Likelihood....................................

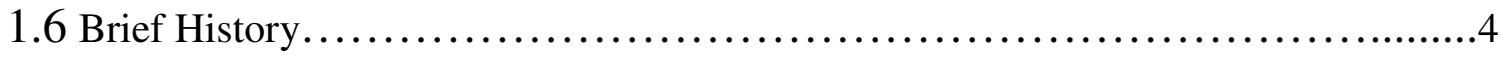

2. INFERENCE PROCEDURE.................................................6

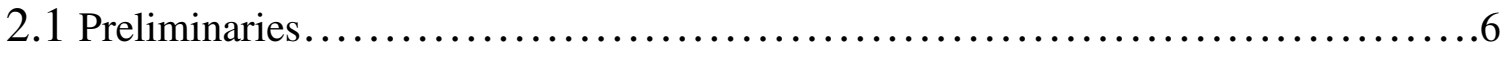

2.2 The JEL confidence region/interval..............................

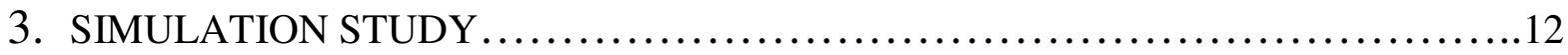

3.1 JEL, EL , and Wald based on Gehan and Kendall estimating equations...........12

3.1.1 Simulation Results and Analysis................................. 14

3.2 Kendall vs. Buckley-James vs.Gehan vs.Logrank Estimator.................. 16

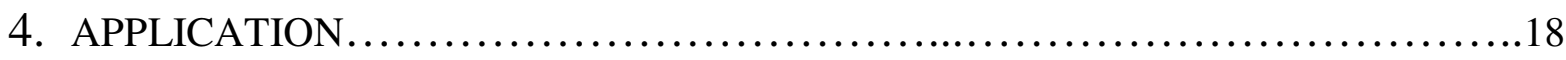

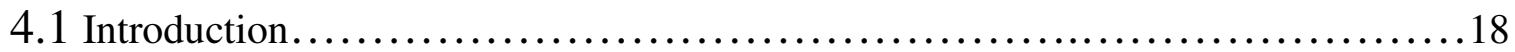




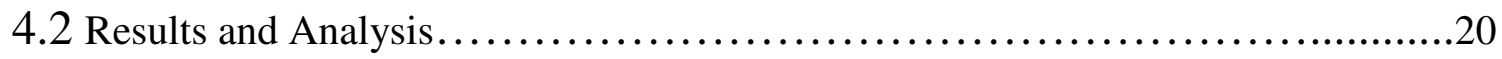

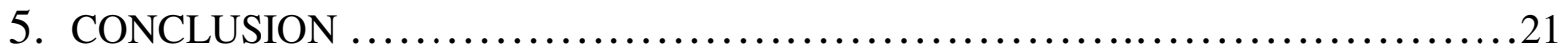

REFERENCES.....................................................22

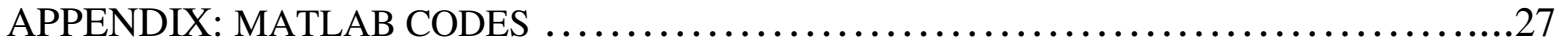




\section{List of Tables}

Table 1: Coverage probability and average length of confidence intervals for the regression parameter

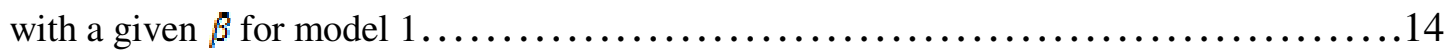

Table 2: Coverage probability and average length of confidence intervals for the regression parameter

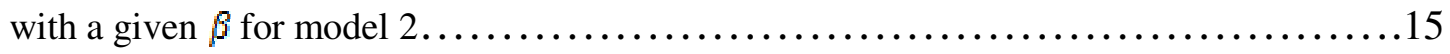

Table 3: Coverage probability using JEL, EL1, B-J, Logrank, and Gehan estimators..............17

Table 4: Confidence intervals using JEL1, JEL2, EL1 and Wald-type procedure ......................20 


\section{Chapter 1}

\section{INTRODUCTION}

\subsection{Accelerated Failure Time Model}

The Cox (1972) proportional hazards model is a popular survival analysis method used to establish a relationship between the covariate and the survival time in censored data. But in many cases, the Cox (1972) proportional hazards model does not always lead to a consistent estimate of the variance and the parameter when the assumptions are not satisfied. To maintain the consistency between the survival time and the covariates, an alternative method called accelerated failure time (AFT) model is quite popularly used. The AFT model assumes that the effect of a covariate is to multiply the predicted event time by some constant. AFT models can be therefore framed as linear models for the logarithm of the survival time. In recent years, many statisticians proposed different estimating methods for the accelerated failure time (AFT); among them, Tsiatis (1990), Ying (1993), and Ritov (1990). These researchers developed an estimating equation based on the linear rank test. Although in theory this method is useful in determining some statistics, it encountered some difficulties when the rank estimation equation is not monotone or continuous. Other researchers such as Lin et al. (1998) developed a rootfinding technique called the linear programming method used to estimate the parameters. To estimate the variance, Tsiatis (1990) developed the nonparametric density function. Wei et al. (1990) also developed a method to estimate a consistent variance under certain conditions. Parzen et al. (1994) estimate the limiting covariance matrices by using a re-sampling method. Although these methods are useful to estimate the variance, they still fail to overcome the under-coverage problem of traditional method. Monotone estimating functions based on Kendall and Gehan estimating equations are used in this thesis. 


\subsection{Monotone Gehan estimating equation}

Fygenson and Ritov (1994) originally developed the rank-based estimating equation for right censoring data. Statisticians such as Lin et al. (1998), inspired by Fygenson and Ritov (1994) estimating equation, used the linear programming technique to find a consistent root. Although the latest method is theoretically useful in some cases, in practice it generally fails to estimate the variance of estimator because the equation is not differentiable. In order to overcome the problems mentioned above, Zhao (2011) proposed EL method. We will apply the jackknife empirical likelihood (JEL) developed by Jing, Yuan, and Zhou (2009) to the monotone Gehan estimating equation used in Zhao (2011) to get a better interval estimation of regression parameters.

\subsection{Kendall estimating equation}

The Kendall's rank regression estimate is defined as follows

$\boldsymbol{U}_{n}(b)=\left\{N\left(\begin{array}{l}n \\ 2\end{array}\right)\right\}^{-1} \sum_{1 \leqq i \epsilon_{j} \leq n} \operatorname{sgn}\left(X_{j}-X_{i}\right) \operatorname{sgn}\left(\varepsilon_{j}(b)-\varepsilon_{i}(b)\right)$

where $\varepsilon_{j}(b)=Y_{j}-\mathrm{b} X_{j}$ and $\mathrm{N}$ is $\sum_{1 \leq i<j \leq n} \operatorname{sgn}\left(X_{j}-X_{i}\right)$.

Kendall estimate is robust against the covariate outliers, as is the Gehan estimating equation, but it is not differentiable in $\beta$ and required a new method to estimate the asymptotic variance of the regression estimate. Lu (2009) developed EL for the AFT model based on Kendall's estimating equation. In this thesis, we will use the jackknife empirical likelihood to construct the confidence regions for the regression parameter based on the Kendall estimating equation.

\subsection{Empirical Likelihood}

Empirical likelihood (EL) method was first introduced by Owen $(1988,1990)$ to determine the shape of the confidence regions without having to estimate the variance. The empirical likelihood does not 
assume a parametric family of distributions for the data. The empirical likelihood method has been extended in different fields such as on the two-sample problems (Liu, Zou and Zhang (2008), Shen and He (2007)), censored median regression model (Zhao and Chen (2008), Zhao and Yang (2008)) etc. For censored linear regression model including AFT models, recent work of EL includes Zhou (2005), Zhao and Huang (2007), and Zhou and Li (2008), Zhao (2011) etc. For a more thorough review of EL before 2001, you may read Owen (2001).

Based on Fygenson and Ritov (1994) estimating equation, Zhao (2011) developed an EL method for the AFT model. Zhao (2011) developed a procedure that avoids the estimation of the variance for normal approximation based method. Motivated by Subramanian (2007), a profile EL for any specified $q$ components of regression parameters is proposed and by using EL, the $4 \chi_{q}^{2}$ limiting distribution of the proposed profile El ratio is obtained accordingly. For more discussions on the EL ratio for $p$-dimension regression analysis, please see Zhao (2011).

\subsection{Jackknife Empirical Likelihood}

Empirical likelihood is very useful in many different occasions, particularly when data subjects to constraints are linear. However, when applied to more complicated statistics such as $U$-statistics, it runs into serious computational difficulties. To overcome these difficulties, Jing, Yuan and Zhou (2009) proposed the jackknife empirical likelihood (JEL) for a $U$-statistic. The method combines two of the popular nonparametric approaches: the jackknife and the empirical likelihood. The key idea of the JEL is to turn the statistic of interest into a sample mean based on jackknife pseudo-values (Quenouille, 1956). If we can show that these pseudo-values are asymptotically independent, we can apply Owen's empirical likelihood for the mean of the jackknife pseudo-values. The most attractive feature with the JEL method is its simplicity, as it is merely a simple application of Owen's empirical likelihood to the "sample" mean 
of jackknife pseudo-values. Theoretically, we will establish Wilks' theorem for one and two-sample $U$ statistics alike. This indicates that the JEL might be potentially useful in handling more general class of statistics than $U$-statistics. Finally, the simulation studies indicate that the JEL compares favorably with other alternatives, and is worthy of serious considerations in statistical inference due to its simplicity.

\subsection{Brief History}

Jackknife empirical likelihood (JEL) is based on both the jackknife and empirical likelihood methods, and can work in rather general settings beyond the simple i.i.d. settings. JEL can also work under weak assumptions so as to make it as widely applicable as possible. JEL works for one and two-sample $U$ statistics. The two samples can be independent but not identically distributed. For other nonlinear statistics, the validity of the JEL has to be checked case by case. The procedure is as follows. For a defined $U$-statistic, we construct a jackknife sample (see, e.g., Shao and Tu (1995)) first, and then treat this jackknife pseudo sample as a sample of i.i.d. observations and apply the standard empirical likelihood method for the mean of i.i.d. observations to obtain the empirical likelihood ratio statistic for the $U$ statistic.

The empirical likelihood method is one of the most famous methodologies for nonparametric statistical inference procedure which has excellent properties. The deployment of empirical likelihood method with respect to survival analysis can be traced back to Thomas and Grunkemeier (1975). The empirical likelihood method was summarized and discussed in Owen $(1988,1990,1991)$, by introducing many great applications and extensions such as constructing nonparametric confidence intervals. Subsequently, Owen and many other statisticians developed this method into a general methodology. 
Jackknife techniques have a long history in statistics. The jackknife method of bias reduction was originally proposed by Quenouille (1956), and then Tukey (1958) subsequently demonstrated how the method could be used to construct a nonparametric estimator of variance. As result, it is often referred to as the Quenouille-Tukey jackknife; see, for example, Efron (1982, p.1). According to Miller (1964, p.1594) the procedure was named the jackknife by Tukey because "a boy scout's jackknife is symbolic of a rough-and-ready instrument capable of being utilized in all contingencies and emergencies." The idea behind the jackknife method of bias reduction is to combine a statistic based on a full sample of data with a set of statistics based on sub-samples in a way that eliminates the first-order bias term from its expectation. The interest is often an estimator of a parameter or parameter vector although functions of model parameters and test statistics can also be considered provided they satisfy (or are assumed to satisfy) certain properties. In the case of a random sample of (i.i.d.) variables the sub-samples are usually obtained by deleting observation $i$ from the full sample. This is sometimes known as the delete-1 jackknife because each sub-sample deletes one observation at a time.

The rest of the thesis is organized as follows. The jackknife empirical likelihood is proposed in Chapter 2 and we also present the procedure related to the methods. Three simulation scenarios performances are presented in Chapter 3: the jackknife empirical likelihood confidence intervals, the empirical likelihood and the traditional normal approximation confidence intervals. We then compare the JEL for Gehan and the Kendall's estimating equations to EL based on Buckley-James, logrank and Gehan estimators, by Zhou (2005) and Zhou and Li (2008). In Chapter 4, we present a real data application. In Chapter 5, a discussion is made and all the technical derivations such as the tables of simulation results and the table of the real application results are presented. The Matlab codes are provided in the Appendix. 


\section{Chapter 2}

\section{INFERENCE PROCEDURE}

\subsection{Preliminaries}

In this sequel, we use the same notations as those in Lu (2009). The setting of the AFT model is as follows. For $i=1, \ldots, n$, let $T_{i}$ be the failure time for $i$ th patient and let $Z_{i}$ 's be the associated $(p \times 1)$ vectors of covariates sequence. $T_{i}>0$, representing the time to event in survival analysis. The AFT model is to relate the regression of the logarithm of survival times, $\log T_{i}$, to their $\mathrm{p}$ covariates through a standard linear regression equations,

$$
\log T_{i}=\beta Z_{i}+\epsilon_{i}, i=1, \ldots, n
$$

where the stochastic errors $\epsilon_{i}$ are independent identically distributed with unknown distribution function $\mathrm{F}$ and the covariate vector $Z_{i}$ is independent of $\varepsilon_{i}$. Since $\mathrm{F}$ is unknown, an estimating equation is a natural approach for estimation and inference on $\beta$. We assume that $C_{i}$ is the censoring times for $T_{i}$. Assuming that $C_{i}$ and $T_{i}$ are independent conditionally on $Z_{i}$, we can only observe $X_{i}=\min \left(T_{i}, C_{i}\right), \Delta_{i}=I\left(T_{i}<C_{i}\right)$ and $Z_{i}, i=1, \ldots, n$, where $I(*)$ is an indicator function. We assume that $\varepsilon_{\mathrm{i}}$ is independent of $\left(C_{i}, Z_{i}\right)$ as was in Fygenson and Ritov (1994). For the regularity conditions, see p.735 of Fygenson and Ritov (1994) and Zhao (2011).

Using the AFT model, we apply the Kendall and Fygenson-Ritov estimating equations to build a confidence region. We use an estimator introduced by Fygenson and Ritov as a solution of a monotone 
estimating equation. Assume that $\beta_{0}$ is the true value of $\beta$, we obtain the following equation $s_{i}(\beta)=\log X_{i}-\beta o^{T} Z_{i}$. The Kendall estimating equation is written as

$$
U(\beta)=n^{-a / 2} \sum_{i=1}^{n} \sum_{j=1}^{n} \operatorname{sgn}\left(Z_{i}-Z_{j}\right) \Delta_{i} I\left\{e_{j}(\beta)>e_{i}(\beta)\right\}
$$

where $\operatorname{sgn}(\cdot)$ is a sign function defined as $\operatorname{sgn}(t)=\left\{\begin{array}{cc}1, & t>0 \\ 0, & t=0 \\ -1, & t<0\end{array}\right.$

The Kendall estimate is robust against outliers (see Heller, 2007). Based on this method, for any fixed $\beta$, $U(\beta)$ becomes a simple $U$-statistic; we can rewrite it as a $U$-statistic with symmetric kernel,

$U(\beta)=n^{-\frac{a}{2}} \sum_{i=1}^{n} \sum_{j=1}^{n} \operatorname{sgn}\left(Z_{i}-Z_{j}\right)\left[\Delta_{i} I\left\{e_{j}(\beta)>e_{i}(\beta)\right]-\Delta_{j} I\left(e_{i}(\beta)>e_{j}(\beta)\right]\right]$

Assume the same conditions as above, the Gehan estimating equation is written as follows

$$
U(\beta)=n^{-3 / 2} \sum_{i=1}^{n} \sum_{j=1}^{n}\left(Z_{i}-Z_{j}\right) \Delta_{i}\left\{\left\{e_{j}(\beta)>e_{i}(\beta)\right\}\right.
$$

Based on this method, for any fixed $\beta, U(\beta)$ is a simple $U$-statistic; we can rewrite it as a $U$-statistic with symmetric kernel,

$V(\beta)=n^{-\frac{3}{2}} \sum_{i=1}^{n} \sum_{j=1}^{n}\left(Z_{i}-Z_{j}\right)\left[\Delta_{i} l\left\{e_{j}(\beta)>e_{i}(\beta)\right]-\Delta_{j} l\left[e_{i}(\beta)>e_{j}(\beta)\right]\right.$

Please refer to Zhao (2011) for more discussions on how to determine the asymptotic variance and the confidence interval of the Wald-type procedure.

As stated in Zhao (2011), although the Wald-type estimation method has its excellent properties, it suffers a serious under-coverage problem for a small sample. Empirical likelihood is therefore used to fix the 
under-coverage problem for linear constraints but when the applications involve nonlinear statistics, EL loses its computational appeals. Jing, Yuan, and Zhou (2009) proposed a new method called jackknife empirical likelihood to overcome the computational burdens.

\subsection{The JEL confidence region/interval}

Let us consider the jackknife empirical likelihood approach in order to make the computation more appealing. By using the Kendall estimating equation, we have the following

$U_{i}=\left(Z_{i}, X_{i}, \Delta_{i}\right)$ and $h\left(U_{i}, U_{j} ; \beta\right)=\operatorname{sgn}\left(Z_{i}-Z_{j}\right)\left\{\Delta_{i} I\left(e_{j}(\beta)>e_{i}(\beta)\right)-\Delta_{j} I\left(e_{i}(\beta)>e_{j}(\beta)\right)\right\} \cdot(7)$

A $U$-statistic of degree 2 with a symmetric kernel $h$ is defined to be

$\boldsymbol{U}_{n}(\beta)=\left(\begin{array}{l}n \\ 2\end{array}\right)^{-1} \sum_{j=1, j=i}^{n}\left\{h\left(U_{i}, U_{j}, \beta\right)\right\}$

Applying the JEL of Jing et al. (2009) to the above equations, we obtain $U_{n}(\beta)=U\left(U_{1}, \ldots, U_{n}\right)$, and the jackknife pseudo-values is defined as

$\hat{V}_{i}=n U_{n}-(n-1) v_{n-1}^{(-i)}, \quad(i=1, \ldots, n)$

where $U_{n-1}^{(-i)}:=U\left(U_{1}, \ldots, U_{i-1}, U_{i+1}, \ldots, U_{n}\right)$, which is obtained from the original data set by removing the ith data value.

Also, similarly, from p.736 of Fygenson and Ritov (1994), $E \vec{V}_{i}=0$, hence the sample mean is as follows $\sum_{i=1}^{n} p_{i} E \hat{v}_{i}=0$.

Furthermore, the jackknife estimator is defined as

$\boldsymbol{U}_{n}=\frac{1}{n} \sum_{i=1}^{n} \hat{V}_{i}$ 
Generally, the jackknife pseudo values are r.v.'s, but asymptotically independent under weak or mild conditions (see Shi, 1984). We can then apply the JEL to the jackknife pseudo values. Let $p=\left(p_{1}, \ldots ., p_{n}\right)$ be a probability vector. Then the empirical likelihood function at the value $\beta$ is given by

$L(\beta)=\max \left\{\prod_{i=1}^{n} p_{i}: \sum_{i=1}^{n} p_{i}=1, \sum_{i=1}^{n} p_{i} \hat{V}_{i}=0, p_{i} \geq 0\right\}$

Note that $\prod_{i=1}^{n} p_{i}$ attains its maximum at $p_{i}=1 / n$. Thus, the jackknife empirical likelihood ratio at $\beta$ is

defined by $\quad R(\beta)=\frac{L(\beta)}{n^{-n}}$

$R(\beta)=\max \left\{\prod_{i=1}^{n} n p_{i}: \sum_{i=1}^{n} P_{i}=1, \sum_{i=1}^{n} p_{i} \hat{V}_{i}=0, p_{i} \geq 0\right\}$

and its logarithm form is

$R(\beta)=\max \left\{\sum_{i=1}^{n} \log \left(n p_{i}\right): \sum_{i=1}^{n} p_{i}=1, \sum_{i=1}^{n} p_{i} \hat{V}_{i}=0, p_{i} \geq 0\right\}$

By using the Lagrange multipliers method,

we have

$p_{i}=\frac{1}{n} \frac{1}{1+\lambda\left(\hat{V}_{i}-E \hat{V}_{i}\right)}$

where $\lambda$ satisfies

$f(\lambda) \equiv \frac{1}{n} \sum_{i=1}^{n} \frac{\hat{V}_{i}-E \hat{V}_{i}}{1+\lambda\left(\hat{V}_{i}-E \hat{V}_{i}\right)}=0$ 
Then, by plugging $p_{i}$ into the logarithm transformation of $R(\beta)$, we obtain

$\bar{\imath}(\beta)=-2 \log R(\beta)=-2 \sum_{i=1}^{n} \log \left(n p_{i}\right)=-2 \sum_{i=1}^{n} \log \left(1+\lambda\left(\hat{V}_{i}\right)\right)$.

The following theorems establish how Wilks' theorem holds and state how the result can be used to construct confidence region for $\beta$.

Theorem 1 Under the above conditions $\hat{l}\left(\beta_{0}\right)$ converges in distribution to $\chi_{p}^{2}$ where $\chi_{p}^{2}$ is a chi-square random variable with $p$ degrees of freedom.

With Theorem 1, an asymptotic $100(1-\alpha) \%$ confidence region for $\beta$ is given by

$R_{E}=\left\{\beta: \ddot{l}\left(\beta_{0}\right) \leq \chi_{p}^{2}(\alpha)\right\}$

where $\chi_{p}^{2}(\alpha)$ is the upper $\alpha$-quantile of the distribution of $\chi_{p}^{2}$

The confidence region for the full set of parameter provides less information in multi-dimensional setting. Regarding the P-values and the confidence intervals for the components of the regression parameters, statisticians make inferences about each element of $\beta$. In this thesis what we want to construct is the EL confidence region for the $\mathrm{q}$ sub-vector $\beta^{(1)}$ of $\beta=\left(\beta^{(1)}{ }_{2} \beta^{(2)}\right)^{\prime}$. Based on Subramanian (2007) profile empirical likelihood for censored median regression models, Zhao (2011) proposed the profile EL for single components by profiling out the nuisance parameters from the full EL. Thus, we will adapt these methods to our settings. 
Define $\beta_{0}=\left(\beta_{0}{ }^{(1)} ; \beta_{0}{ }^{(2)}\right)$ and $\mathcal{N}^{\prime}=\left\{\beta^{(2)} ;\left\|\beta^{(2) \prime}-\hat{\beta}^{(2) !}\right\|=O\left(n^{-1 / 3}\right)\right\}$. The profile EL ratio at $\beta^{(1)}$ is defined as $\tilde{l}_{\text {Profibe }}\left(\beta^{(1)}\right)=\min _{\beta^{(2)} \in \mathcal{N}} \hat{l}\left(\left(\beta^{(1)} ; \beta^{(2)}\right)\right)$. The corresponding theorem for the full EL is therefore obtained.

Theorem 2: Under the above conditions, $\tilde{\boldsymbol{l}}_{\text {Profin }}\left(\beta_{0}^{(1)}\right)$ converges in distribution to $\chi_{q}^{2}$, where $\chi_{q}^{2}$ is a chisquare random variable with q degrees of freedom.

Using this Theorem, an asymptotic $100(1-\alpha) \%$ confidence region for $\beta^{(1)}$ is given by

$R_{p}=\left\{\beta_{1}^{(1)}, \hat{l}_{p_{r o f i l}}\left(\beta^{(1)}\right) \leq \chi_{q}^{2}(\alpha)\right\}$

where $\chi_{q}^{2}(\alpha)$ is the upper $\alpha$-quintile of the distribution of $\chi_{q}^{2}$.

For Fygenson-Ritov estimating equation, similarly we can obtain the profile jackknife EL $\hat{\mathbb{Z}}_{\text {profile }}\left(\beta_{0}^{(1)}\right)$ by eliminating the nuisance parameters from the full JEL. The resulting JEL confidence intervals for $\beta_{0}^{(1)}$ is obtained, which is consistent with Theorem 1 and Theorem 2. 


\section{Chapter 3}

\section{SIMULATION STUDY}

\subsection{JEL, and EL vs. Wald-type based on Gehan and Kendall estimating equations}

Based on the Gehan and Kendall estimating equations, extensive simulation studies are conducted to compare the performances of the confidence intervals of jackknife empirical likelihood, empirical likelihood method and the Wald-type based approach. The performances of the proposed procedures are compared in terms of coverage probability and average length of confidence intervals in different settings. We use the same settings as those in $\mathrm{Lu}(2009)$.

Assuming there are only one covariate $\mathrm{Z}$ and a true parameter $\beta_{0}=2$, skewed error distribution and the symmetric error distribution are the two models considered to conduct the simulation runs. Model 1 has a covariate $Z$ and is uniformly distributed in $[-1,1]$. The censoring time $\mathrm{C}$ follows uniform distribution in $[0, \mathrm{c}]$, where $\mathrm{c}$ controls the censoring rate. The error term has a standard Gumbel distribution when $\mu=0$ and $\beta=1$, and the cumulative distribution function skewed to the right is defined as follows

$F(x)=e^{-e^{-x}}$

We generated the error term as the following $\epsilon_{i}=\log (-\log (U))$, where $U$ is a uniform variable in $[0,1]$. Thus, the survival time can be obtained by $T_{i}=\exp \left(\beta_{0} Z_{i}+\epsilon_{i}\right)$, where $\beta_{0}=2$.

Model 2 has a covariate $\mathrm{Z}$ and is uniformly distributed in $[0.5,1.5]$. The censoring time $\mathrm{C}$ is distributed as $2 \exp (1)+c$, where $\exp (1)$ is a standard exponential distribution, and c controls the censoring rate. The symmetric error distribution is similar to that of the standard Normal distribution $\mathbf{N}(0,1)$. 
The censoring time $\mathrm{C}$ can be generated as follows $C=-2 \log (\boldsymbol{U})+c$, where $U$ is a uniform variable in $[0,1]$ and $\mathrm{c}$ is a constant.

Assuming a true value $\beta_{0}=2$, four different censoring rates with approximately $15 \%, 30 \%$ and $45 \%$, and $60 \%$ respectively, which represent light censoring, medium censoring and moderate heavy censoring, and very heavy censoring rate. The sample sizes are 30, 50, 75 and 100, representing very small, relatively small, moderate and large samples respectively. Therefore, we have 16 data settings in total for each of the two models. Each data set is simulated 10000 times and the results are displayed in Table 1 and Table 2.

Table 1 and Table 2 displayed the results of the Wald-type, the empirical likelihood, and the jackknife empirical likelihood methods. The censoring rates are approximately $15 \%, 30 \%, 45 \%$, and the sample size, based on 10,000 simulated data sets, is $30,50,75$, and 100 . The three methods have better performances in term of coverage probabilities and average lengths when the total sample size increases. The coverage probability for large sample, that is $n=100$, works well with right coverage probability of 90\%, 95\%. The Wald-type method has greater under-coverage when the sample size is small, while the empirical likelihood and the jackknife empirical likelihood methods have a better coverage probability for all the nominal levels. The three methods have a better accuracy of the coverage probabilities when the censoring rate decreases because there are fewer information losses. 
Table 1: Coverage probability and average lengths of confidence intervals for the regression parameter $\beta$ with model 1.

\begin{tabular}{|c|c|c|c|c|c|c|c|c|c|c|}
\hline \multirow[b]{2}{*}{ CR } & \multirow[b]{2}{*}{$n$} & \multirow[b]{3}{*}{ Coverage } & \multirow[b]{2}{*}{ Wald } & \multicolumn{3}{|c|}{$1-\alpha=0.90$} & \multicolumn{3}{|c|}{$1-\alpha=0.95$} & \multirow[b]{2}{*}{ JEL2 } \\
\hline & & & & EL1 & JEL1 & JEL2 & Wald & EL1 & JEL1 & \\
\hline & 30 & & 0.8691 & 0.8992 & 0.8997 & 0.8989 & 0.9228 & 0.9436 & 0.9489 & 0.9478 \\
\hline \multirow{7}{*}{$15 \%$} & & Length & 1.4349 & 1.5739 & 1.5741 & 1.5738 & 1.7101 & 1.9037 & 1.9056 & 1.9051 \\
\hline & 50 & Coverage & 0.8862 & 0.9087 & 0.9098 & 0.9089 & 0.9342 & 0.9524 & 0.9571 & 0.9562 \\
\hline & & Length & 1.0859 & 1.1844 & 1.1848 & 1.1851 & 1.2943 & 1.4212 & 1.4227 & 1.4223 \\
\hline & 75 & Coverage & 0.8889 & 0.9134 & 0.9198 & 0.9189 & 0.9411 & 0.9592 & 0.9598 & 0.9593 \\
\hline & & Length & 0.8735 & 0.9515 & 0.9532 & 0.9527 & 1.0412 & 1.1422 & 1.1431 & 1.1428 \\
\hline & 100 & Coverage & 0.8941 & 0.9157 & 0.9176 & 0.9163 & 0.9429 & 0.9617 & 0.9669 & 0.9658 \\
\hline & & Length & 0.7517 & 0.8073 & 0.8086 & 0.8083 & 0.8965 & 0.9783 & 0.9799 & 0.9793 \\
\hline \multirow{8}{*}{$30 \%$} & 30 & Coverage & 0.8676 & 0.8953 & 0.8991 & 0.8979 & 0.9168 & 0.9369 & 0.9476 & 0.9468 \\
\hline & & Length & 1.6869 & 1.8169 & 1.8183 & 1.8177 & 2.0109 & 2.1744 & 2.1753 & 2.1748 \\
\hline & 50 & Coverage & 0.8769 & 0.8987 & 0.8998 & 0.8989 & 0.9276 & 0.9457 & 0.9489 & 0.9481 \\
\hline & & Length & 1.2683 & 1.3631 & 1.3645 & 1.3639 & 1.5114 & 1.6262 & 1.6276 & 1.6261 \\
\hline & 75 & Coverage & 0.8836 & 0.9067 & 0.9103 & 0.9098 & 0.9375 & 0.9521 & 0.9581 & 0.9573 \\
\hline & & Length & 1.0223 & 1.1047 & 1.1053 & 1.1049 & 1.2169 & 1.3139 & 1.3153 & 1.3148 \\
\hline & 100 & Coverage & 0.8919 & 0.9117 & 0.9189 & 0.9179 & 0.9422 & 0.9596 & 0.9617 & 0.9609 \\
\hline & & Length & 0.8816 & 0.9469 & 0.9478 & 0.9471 & 1.0488 & 1.1363 & 1.1404 & 1.1401 \\
\hline \multirow{8}{*}{$45 \%$} & 30 & Coverage & 0.8497 & 0.8729 & 0.8789 & 0.8778 & 0.9087 & 0.9194 & 0.9215 & 0.9208 \\
\hline & & Length & 2.0332 & 2.1771 & 2.1789 & 2.1785 & 2.4218 & 2.5978 & 2.6012 & 2.6007 \\
\hline & 50 & Coverage & 0.8708 & 0.8852 & 0.8971 & 0.8963 & 0.9238 & 0.9337 & 0.9399 & 0.9388 \\
\hline & & Length & 1.5253 & 1.5963 & 1.6617 & 1.6609 & 1.8163 & 1.9011 & 1.9084 & 1.9079 \\
\hline & 75 & Coverage & 0.8805 & 0.9011 & 0.9089 & 0.9081 & 0.9342 & 0.9441 & 0.9488 & 0.9479 \\
\hline & & Length & 1.2298 & 1.2965 & 1.2974 & 1.2969 & 1.4651 & 1.5283 & 1.5291 & 1.5286 \\
\hline & 100 & Coverage & 0.8876 & 0.9046 & 0.9089 & 0.9077 & 0.9399 & 0.9478 & 0.9518 & 0.9512 \\
\hline & & Length & 1.0521 & 1.1223 & 1.0370 & 1.0369 & 1.2523 & 1.3241 & 1.1897 & 1.1895 \\
\hline \multirow{8}{*}{$60 \%$} & 30 & Coverage & 0.8142 & 0.8386 & 0.8678 & 0.8669 & 0.8763 & 0.8868 & 0.8991 & 0.8983 \\
\hline & & Length & 2.6108 & 2.7791 & 2.8441 & 2.8438 & 3.1101 & 3.2618 & 3.3685 & 3.3681 \\
\hline & 50 & Coverage & 0.8489 & 0.8494 & 0.8789 & 0.8781 & 0.9013 & 0.9034 & 0.9211 & 0.9203 \\
\hline & & Length & 1.9466 & 1.9872 & 2.1032 & 2.1028 & 2.3187 & 2.3193 & 2.4893 & 2.4891 \\
\hline & 75 & Coverage & 0.8712 & 0.8673 & 0.8827 & 0.8821 & 0.9219 & 0.9167 & 0.9376 & 0.9371 \\
\hline & & Length & 1.5722 & 1.5893 & 1.6645 & 1.6639 & 1.8744 & 1.8752 & 1.8898 & 1.8591 \\
\hline & 100 & Coverage & 0.8744 & 0.8816 & 0.8975 & 0.8968 & 0.9289 & 0.9279 & 0.9432 & 0.9428 \\
\hline & & Length & 1.3365 & 1.3826 & 1.4321 & 1.4316 & 1.6032 & 1.6217 & 1.7127 & 1.7118 \\
\hline
\end{tabular}

CR: censoring rate

EL1: empirical likelihood using Kendall estimating equation

JEL1: jackknife empirical likelihood using Kendall estimating equation

JEL2: jackknife empirical likelihood using Gehan estimating equation 
Table 2: Coverage probability and average lengths of confidence intervals for the regression

parameter $\beta$ with model 2

\begin{tabular}{|c|c|c|c|c|c|c|c|c|c|c|}
\hline \multirow[b]{2}{*}{ CR } & \multirow[b]{2}{*}{$\mathrm{n}$} & \multirow[b]{3}{*}{ Compros } & \multirow[b]{2}{*}{ Wald } & \multicolumn{4}{|c|}{$1-\alpha=0.90$} & \multicolumn{3}{|c|}{$1-\alpha=0.95$} \\
\hline & & & & EL1 & JEL1 & JEL2 & Wald & EL1 & JEL1 & JEL2 \\
\hline & 30 & & 0.8543 & 0.9086 & 0.9113 & 0.9107 & 0.9126 & 0.9511 & 0.9609 & 0.9598 \\
\hline \multirow{7}{*}{$15 \%$} & & Length & 2.3071 & 2.4428 & 2.5142 & 2.5133 & 2.7488 & 2.8873 & 2.9251 & 2.8898 \\
\hline & 50 & Coverage & 0.8758 & 0.9165 & 0.9192 & 0.9183 & 0.9297 & 0.9614 & 0.9689 & 0.9679 \\
\hline & & Length & 1.7436 & 1.8877 & 1.8886 & 1.8878 & 2.0776 & 2.2515 & 2.2569 & 2.2563 \\
\hline & 75 & Coverage & 0.8856 & 0.9184 & 0.9196 & 0.9189 & 0.9379 & 0.9632 & 0.9667 & 0.9658 \\
\hline & & Length & 1.4098 & 1.5139 & 1.5147 & 1.5143 & 1.6798 & 1.8265 & 1.8289 & 1.8283 \\
\hline & 100 & Coverage & 0.8884 & 0.9124 & 0.9202 & 0.9193 & 0.9419 & 0.9632 & 0.9688 & 0.9682 \\
\hline & & Length & 1.2162 & 1.2854 & 1.3051 & 1.2984 & 1.4492 & 1.5598 & 1.6012 & 1.6007 \\
\hline \multirow{8}{*}{$30 \%$} & 30 & Coverage & 0.8526 & 0.9006 & 0.9117 & 0.9111 & 0.9069 & 0.9462 & 0.9512 & 0.9504 \\
\hline & & Length & 2.4351 & 2.5434 & 2.6012 & 2.6006 & 2.9013 & 2.9931 & 3.1241 & 3.1232 \\
\hline & 50 & Coverage & 0.8764 & 0.9064 & 0.9114 & 0.9109 & 0.9239 & 0.9519 & 0.9627 & 0.9621 \\
\hline & & Length & 1.8432 & 1.9751 & 2.1131 & 2.1127 & 2.1966 & 2.3565 & 2.4126 & 2.4122 \\
\hline & 75 & Coverage & 0.8831 & 0.9127 & 0.9184 & 0.9179 & 0.9379 & 0.9616 & 0.9669 & 0.9662 \\
\hline & & Length & 1.4887 & 1.5897 & 1.6117 & 1.6113 & 1.7736 & 1.9086 & 1.9125 & 1.9122 \\
\hline & 100 & Coverage & 0.8834 & 0.9092 & 0.9124 & 0.9121 & 0.9383 & 0.9588 & 0.9601 & 0.9598 \\
\hline & & Length & 1.2811 & 1.3529 & 1.3613 & 1.3611 & 1.5259 & 1.6345 & 1.7112 & 1.7109 \\
\hline \multirow{8}{*}{$45 \%$} & 30 & Coverage & 0.8441 & 0.8838 & 0.8991 & 0.8983 & 0.8991 & 0.9333 & 0.9464 & 0.9458 \\
\hline & & Length & 2.6694 & 2.8715 & 2.9254 & 2.9249 & 3.1811 & 3.4432 & 3.5213 & 3.5203 \\
\hline & 50 & Coverage & 0.8693 & 0.8965 & 0.9013 & 0.9007 & 0.9211 & 0.9419 & 0.9512 & 0.9503 \\
\hline & & Length & 2.0311 & 2.1351 & 2.2354 & 2.2349 & 2.4198 & 2.5534 & 2.6232 & 2.6228 \\
\hline & 75 & Coverage & 0.8807 & 0.9081 & 0.9109 & 0.9102 & 0.9331 & 0.9498 & 0.9522 & 0.9515 \\
\hline & & Length & 1.6303 & 1.7278 & 1.7892 & 1.7885 & 1.9422 & 2.0531 & 2.6211 & 2.6207 \\
\hline & 100 & Coverage & 0.8878 & 0.9079 & 0.9126 & 0.9121 & 0.9369 & 0.9545 & 0.9598 & 0.9591 \\
\hline & & Length & 1.4075 & 1.4886 & 1.5125 & 1.5122 & 1.6771 & 1.7754 & 1.7768 & 1.7761 \\
\hline \multirow{8}{*}{$60 \%$} & 30 & Coverage & 0.8322 & 0.8636 & 0.8988 & 0.8982 & 0.8871 & 0.9095 & 0.9371 & 0.9362 \\
\hline & & Length & 3.0165 & 3.1971 & 3.4178 & 3.4171 & 3.5937 & 3.7969 & 3.8112 & 3.8103 \\
\hline & 50 & Coverage & 0.8708 & 0.8785 & 0.8996 & 0.8991 & 0.9181 & 0.9224 & 0.9463 & 0.9456 \\
\hline & & Length & 2.2773 & 2.3437 & 2.4214 & 2.4206 & 2.7134 & 2.7911 & 2.8321 & 2.8315 \\
\hline & 75 & Coverage & 0.8820 & 0.8946 & 0.9087 & 0.9078 & 0.9305 & 0.9398 & 0.9512 & 0.9508 \\
\hline & & Length & 1.8234 & 1.8976 & 1.9214 & 1.9208 & 2.1725 & 2.2440 & 2.4175 & 2.4171 \\
\hline & 100 & Coverage & 0.8811 & 0.8996 & 0.9021 & 0.9017 & 0.9332 & 0.9424 & 0.9556 & 0.9549 \\
\hline & & Length & 1.5664 & 1.6454 & 1.7231 & 1.7227 & 1.8663 & 1.9424 & 2.1231 & 2.1226 \\
\hline
\end{tabular}


The Wald-type procedure has a slightly shorter average length compared to the empirical likelihood and the jackknife empirical likelihood methods; thus, the shorter the average length of confidence interval, the better the confidence interval. Also we notice that when sample size increases, the average length shortens and the censoring rate decreases and the reason is the larger the sample size, the less information is susceptible to be lost. The Wald type confidence interval is symmetric; however the empirical likelihood and the jackknife empirical likelihood confidence intervals are not symmetric because EL1 and JEL confidence intervals are built through their data set instead of a given distribution. In term of coverage probability, JEL1 is better than JEL2 which is also better than EL1 but in terms of average length, the Wald type method has a slightly shorter length than JEL1 and JEL2.

\subsection{Kendall vs. Buckley-James vs. Gehan vs. Logrank Estimator}

Jackknife empirical likelihood method is used to compare Kendall's Tau, Buckley-James (Zhou and Li , 2008) and Logrank estimators (Zhou, 2005) in terms of coverage probability and average length. In this regard, model 3 is introduced with a covariate $Z$ and the error term follows a Normal distribution in $N$ $\left(1,0.5^{2}\right)$. We use the same settings as those in $\mathrm{Lu}(2009)$.

The censoring time $\mathrm{C}$ also follows a Normal distribution in $N\left(\mu, 4^{2}\right)$, where $\mu=-1.8,1,3.1$ and 6.1 respectively and censoring rates are $10 \%, 30 \%, 50 \%, 75 \%$. The sample size $\mathrm{n}$ is 50,100 and 200 . The coverage probabilities are based on 5000 simulation repetitions. Considering $\beta_{0}=1$

we can observe $\mathrm{X}_{i}=\min \left(\mathrm{Y}_{i}, \mathrm{C}_{i}\right), \Delta_{i}=\mathrm{I}\left(\mathrm{Y}_{i} \leq \mathrm{C}_{i}\right)$

and $Z_{i}, i=1, \ldots, n$, where $($.$) is an indicator function,$

the AFT model is defined as

$$
Y_{i}=\beta Z_{i}+\epsilon_{i}, \quad i=1, \ldots, n
$$


Table 3: Coverage probability using JEL, EL1, B-J, Logrank, and Gehan estimators

\begin{tabular}{|c|c|c|c|c|c|c|c|c|c|c|c|c|c|}
\hline $\begin{array}{c}\text { Censoring } \\
\text { rate }\end{array}$ & $\mathrm{n}$ & B-J & Logrank & Gehan & EL1 & JEL1 & JEL2 & B-J & Logrank & Gehan & EL1 & JEL1 & JEL2 \\
\hline \multirow[b]{2}{*}{$10 \%$} & 50 & 0.8932 & 0.8885 & 0.8844 & 0.9125 & 0.9179 & 0.9163 & 0.9417 & 0.9411 & 0.9367 & 0.9522 & 0.9559 & 0.9546 \\
\hline & 100 & 0.8893 & 0.8918 & 0.8916 & 0.9234 & 0.9283 & 0.9274 & 0.9419 & 0.9491 & 0.9452 & 0.9632 & 0.9678 & 0.9664 \\
\hline \multirow[t]{2}{*}{$30 \%$} & 100 & 0.8943 & 0.8893 & 0.8879 & 0.9221 & 0.9271 & 0.9279 & 0.9481 & 0.9422 & 0.9392 & 0.9569 & 0.9592 & 0.9581 \\
\hline & 200 & 0.8934 & 0.9147 & 0.8967 & 0.9116 & 0.9162 & 0.9149 & 0.9479 & 0.9631 & 0.9453 & 0.9582 & 0.9594 & 0.9585 \\
\hline $50 \%$ & 50 & 0.8843 & 0.8807 & 0.8657 & 0.8981 & 0.8998 & 0.8987 & 0.9332 & 0.9329 & 0.9234 & 0.9372 & 0.9426 & 0.9414 \\
\hline \multirow[t]{2}{*}{$75 \%$} & 100 & 0.8824 & 0.8747 & 0.8548 & 0.8863 & 0.8981 & 0.8972 & 0.9352 & 0.9311 & 0.9125 & 0.9345 & 0.9415 & 0.9403 \\
\hline & 200 & 0.8935 & 0.8865 & 0.8776 & 0.9027 & 0.9073 & 0.9058 & 0.9447 & 0.9448 & 0.9366 & 0.9497 & 0.9538 & 0.9528 \\
\hline
\end{tabular}

B-J: EL for Buckley-James estimator by Zhou and Li (2008) Logrank: EL for logrank estimator by Zhou (2005)

Gehan: EL for Gehan estimator by Zhou (2005)

JEL1: Jackknife empirical likelihood using Kendall estimating equation by Kendall (1938)

JEL2: Jackknife empirical likelihood using Gehan estimating equation by Fygenson and Ritov (1994)

The results displayed in Table 3 show that jackknife empirical likelihood using Kendall estimating equation (JEL1) has a better performance in terms of coverage probability, which is followed closely by jackknife empirical likelihood using Gehan equation (JEL2). In most cases, Kendall's coverage probability (EL1) has a better performance compared to Buckley-James, Gehan, and Logrank estimators; for smaller sample size, Kendall outperforms the three estimators. Gehan has the worst performance coverage among the different methods. In conclusion, JEL1 and JEL2 have similar coverage probability and are better than Logrank, B-J and EL1 estimators. 


\title{
Chapter 4
}

\begin{abstract}
APPLICATION

\subsection{Introduction}

In this section, we apply jackknife empirical likelihood methods to bone marrow transplant procedure described by Klein and Maeschberger (1997) in "survival analysis: techniques for censored and truncated data”. Lu (2009) also used EL for Kendall's estimating equation to analyze this dataset. We combine these results together.
\end{abstract}

The preparative regimen used in this study of allogeneic marrow transplants for patients with acute myeloctic leukemia (AML) and acute lymphoblastic leukemia (ALL) was a combination of $16 \mathrm{mg} / \mathrm{kg}$ of oral Busulfan (BU) and $120 \mathrm{mg} / \mathrm{kg}$ of intravenous cyclophosphamide (Cy). A total of 137 patients (99 AML, 38 ALL) were treated at one of four hospitals: 76 at The Ohio State University Hospitals (OSU) in Columbus; 21 at Hahnemann University (HU) in Philadelphia; 23 at St. Vincent's Hospital (SVH) in Sydney Australia; and 17 at Alfred Hospital (AH) in Melbourne. The study consists of transplants conducted at these institutions from March 1, 1984, to June 30, 1989. The maximum follow-up was 7 years. There were 42 patients who relapsed and 41 who died while in remission. Twenty-six patients had an episode of acute GVHD, and 17 patients either relapsed or died in remission without their platelets returning to normal levels.

Several potential risk factors were measured at the time of transplantation. For each disease, patients were grouped into risk categories based on their status at the time of transplantation. These 
categories were as follows: ALL (38 patients), AML low-risk first remission (54 patients), and AML high-risk second remission or untreated first relapse (15 patients) or second or greater relapse or never in remission (30 patients). Other risk factors measured at the time of transplantation included recipient and donor gender (80 and 88 males respectively), recipient and donor cytomegalovirus immune status (CMV) status (68 and 58 positive, respectively), recipient and donor age (ranges 7-52 and 2-56, respectively), waiting time from diagnosis to transplantation (range 0.8-87.2 months, mean 19.7 months), and, for AML patients, their French-American-British (FAB) classification based on standard morphological criteria. AML patients with an FAB classification of M4 or M5 (45/99 patients) were considered to have a possible elevated risk of relapse or treatment-related death.

The censoring rate used in this simulation study is $40.88 \%$ and the model is defined as follows $\log T_{i}=\beta Z_{i}+\epsilon_{i}$, where $T_{i}$ is Time to Death, and $Z_{i}$ is the covariate and $\epsilon_{\mathrm{i}}$ is the error term .

Four different coefficient estimates $\beta$ and a covariate are used to construct the confidence intervals. As Table 4 shows, among the covariates, FAB is very significant across all the nominal levels when it is associated to the Wald-type procedure. Among the other three coefficient estimates, covariate called Group is significant at confidence level 0.90. As in Lu (2009), the variable Age is a scaled interaction between patient Age and donor Age, and is defined as Age $=($ patient Age -28$) \times($ donor Age -28) /100. Age is significant at several confidence levels. Lastly, we notice that the Waiting time is not significant at any confidence levels as shown in Table 4. JEL1 and JEL2 have longer lengths; therefore, their confidence intervals are longer, which is in phase with the results we found in our simulation study. 


\subsection{Results and Analysis}

Table 4: Confidence intervals using JEL1, JEL2, EL1 and Wald-type procedure

\begin{tabular}{|c|c|c|c|c|c|c|c|c|c|}
\hline \multirow{9}{*}{$\frac{\text { D }}{3}$} & & \multirow{2}{*}{\multicolumn{2}{|c|}{$\frac{\text { FAB }}{-0.8388}$}} & \multicolumn{2}{|c|}{ Group } & \multicolumn{2}{|c|}{ Age } & \multicolumn{2}{|c|}{ TimeToTrx } \\
\hline & $\beta$ & & & \multicolumn{2}{|c|}{-0.4558} & \multicolumn{2}{|c|}{-0.4588} & \multicolumn{2}{|c|}{-0.2055} \\
\hline & & \multicolumn{2}{|r|}{$\mathrm{CI}$} & \multicolumn{2}{|c|}{$\mathrm{CI}$} & \multicolumn{2}{|r|}{$\mathrm{CI}$} & \multicolumn{2}{|c|}{$\mathrm{CI}$} \\
\hline & 0.90 & $(-1.3483$ & $-0.3292)$ & $(-0.8418$ & $-0.071)$ & $(-0.7791$ & $0.1408)$ & $(-0.4382$ & $0.0271)$ \\
\hline & Length & \multicolumn{2}{|c|}{1.0191} & \multicolumn{2}{|c|}{0.7708} & \multicolumn{2}{|c|}{0.6383} & \multicolumn{2}{|c|}{0.4653} \\
\hline & 0.95 & $(-1.4463$ & $-0.2317)$ & $(-0.9157$ & $0.0042)$ & $(-0.8381$ & $-0.0799)$ & $(-0.4826$ & $0.0715)$ \\
\hline & Length & \multicolumn{2}{|r|}{1.2146} & \multicolumn{2}{|c|}{0.9199} & \multicolumn{2}{|c|}{0.7582} & \multicolumn{2}{|c|}{0.5541} \\
\hline & 0.99 & $(-1.6372$ & $-0.0409)$ & $(-1.0602$ & $0.1487)$ & $(-0.9574$ & 0.0396) & $(-0.5697$ & $0.1585)$ \\
\hline & Length & \multicolumn{2}{|r|}{1.5963} & \multicolumn{2}{|c|}{1.2089} & \multicolumn{2}{|c|}{0.997} & \multicolumn{2}{|c|}{0.7282} \\
\hline \multirow{6}{*}{$\overrightarrow{3}$} & 0.90 & $(-1.3728$ & $-0.2543)$ & $(-0.8629$ & $-0.0385)$ & $(-0.9321$ & $-0.1722)$ & $(-0.4908$ & -0.0136 \\
\hline & Length & & 1.1185 & \multicolumn{2}{|c|}{0.8244} & \multicolumn{2}{|c|}{0.7599} & \multicolumn{2}{|c|}{0.4772} \\
\hline & 0.95 & $(-1.4936$ & $-0.1244)$ & $(-0.9445$ & $0.0421)$ & $(-1.0505$ & $-0.1089)$ & $(-0.5731$ & $0.0214)$ \\
\hline & Length & & 1.3692 & \multicolumn{2}{|c|}{0.9866} & \multicolumn{2}{|c|}{0.9416} & \multicolumn{2}{|c|}{0.5945} \\
\hline & 0.99 & $(-1.6192$ & $0.1329)$ & $(-1.1252$ & $0.2236)$ & $(-1.2551$ & $0.0057)$ & $(-0.7335$ & $0.0777)$ \\
\hline & Length & & 1.7521 & & 488 & & 608 & & 12 \\
\hline & 0.90 & $(-1.4972$ & $-0.2942)$ & $(-0.9275$ & $-0.0369)$ & $(-1.0262$ & -0.1843 & $(-0.5308$ & $-0.0214)$ \\
\hline & Length & & 1.2030 & & 906 & & 419 & & 994 \\
\hline & 0.95 & $(-1.5238$ & $-0.1348)$ & $(-1.1563$ & $0.0572)$ & $(-1.3081$ & $-0.1068)$ & $(-0.6339$ & $0.0172)$ \\
\hline 3 & Length & & 1.3890 & & 135 & & 013 & & 511 \\
\hline 武 & 0.99 & $(-1.7278$ & $0.1483)$ & $(-1.2457$ & $0.3189)$ & $(-1.3363$ & $0.0198)$ & $(-0.9663$ & $0.0776)$ \\
\hline & Length & & 1.8761 & & 646 & & 561 & & 139 \\
\hline & 0.90 & $(-1.4915$ & $-0.2833)$ & $(-0.9279$ & $-0.0198)$ & $(-0.9878$ & $-0.1586)$ & $(-0.5425$ & $-0.0198)$ \\
\hline & Length & & 1.2083 & & 081 & & 292 & & 227 \\
\hline & 0.95 & $(-1.5242$ & $-0.1353)$ & $(-1.1558$ & $0.0395)$ & $(-1.0836$ & $-0.1099)$ & $(-0.6428$ & $0.0259)$ \\
\hline ก & Length & & 1.3889 & & 953 & & 935 & & 687 \\
\hline 武 & 0.99 & $(-1.7365$ & $0.1379)$ & $(-1.2265$ & $0.3142)$ & $(-1.4006$ & $0.0126)$ & $(-0.9789$ & $0.0756)$ \\
\hline & Length & & 1.8744 & & 407 & & 132 & & 45 \\
\hline
\end{tabular}




\section{Chapter 5}

\section{CONCLUSION}

The recommended coverage probability is the one that that is close enough to the nominal level and the best average length is the shortest one. In terms of probability coverage, JEL1 and JEL2 are close to their corresponding nominal levels. In addition, when the sample sizes are large the coverage probabilities are more accurate and the average lengths are the shortest. The jackknife empirical likelihood using Kendall estimating equation outperformed the EL1 method and the Wald-type procedure. In terms of average length, Wald procedure is the best. We also notice that JEL1 and JEL2 have better coverage probability. When the sample size increases, all the proposed methods have better performances in terms of coverage probabilities and average lengths; However for smaller sample sizes, JEL1 and JEL2 are the best. In addition, it takes less time to compute JEL1 and JEL2. Zhao (2011) proposed EL method to fix the under-coverage problem that is presented in the Wald-type procedure and this method worked well in our simulation study as well. By using JEL1 and JEL2 to simulate the data set, we noticed that for larger sample size, there is over-coverage problem that needs to be addressed. In our future work, we will investigate the use of bootstrap calibration using JEL to fix the over-coverage problem. 


\section{REFERNCES}

[1] Andrews, D. F. and Herzberg, A. M., Data: A Collection of Problems from Many fields for the student and Research Worker., Springer, 1985.

[2]Arvesen, J. N., Jackknifing U-statistics, Annals of Mathematical Statistics, Vol. 40, pp. 2076-2100, 1969.

[3] Efron, B., The jackknife, the bootstrap and other resampling plans, Society of Industrial and Applied Mathematics CBMS-NSF Monographs, Vol. 38, pp.1, 1982.

[4] Chen, J., Mulayath, A. and Bovas, A., Adjusted empirical likelihood and its properties, Journal of Computational and Graphical Statistics, Vol. 17, pp. 426-443, 2008.

[5] Chen, S.X., Empirical likelihood confidence intervals for nonparametric density estimation, Biometrika, Vol 83, pp. 329-341, 1986.

[6] Cox, D.R., Regression models and life-tables (with discussion), J.R. Statist. Soc. B., Vol. 34, pp.187$220,1972$.

[7] Fygenson,M. and Ritov,Y., Monotone estimating equations for censored data, Ann. Math. Statistics, Vol. 22, pp. 732-746, 1994.

[8] Halmos, P. R., The theory of unbiased estimation, Annals of Mathematical Statistics, Vol. 17, pp. 3443, 1946. 
[9] Heller, G., Smoothed rank regression with censored data, J. Amer. Statist. Assoc., Vol. 102, pp.552559, 2007.

[10] Hoeding, W., A class of statistics with asymptotically normal distribution, Annals of Mathematical Statistics,Vol. 19, pp. 293-325, 1948.

[11] Jing, B. Y., Yuan, J. Q. and Zhou, W., Jackknife empirical likelihood, Journal of the American Statistical Association, Vol. 104, pp. 1224-1232, 2009.

[12] Klein, J.P. and Maoeschberger, M.L., Survival analysis techniques for censored and truncated data, $2^{\text {nd }}$ edition, Springer, 1997.

[13] Koroljuk, V. S. and Borovskich, Yu. V., Theory of U-statistics, Mathematics and Its Applications, Vol. 273, Kluwer Academic Publishers, 1994.

[14] Lee, A. J., U-statistics, Theory and Practice, New York: Marcel Dekker,Inc., 1990.

[15] Li, Y. and Wang, Q.H., Empirical likelihood regression analysis for right censored data, Statist. Sinica, Vol. 13, pp. 51-68, 2003.

[16] Lin et al., Accelerated failure time models for counting processes, Biometrika, Vol. 85, pp. 605-618, 1998.

[17] Lin,D.Y., and Geyer, C.J., Computational methods for semi-parametric linear regression with censored data, J.Comp.Graph.Statist.,Vol 1, pp.77-90, 1992.

[18] Liu,Y., Zou,C., and Zhang, R., Empirical likelihood for the two-sample mean problem, Statistics and Probability Letters, Vol. 78, pp. 548-556, 2008. 
[19] Lu, Y., Empirical likelihood inference for the accelerated failure time model via Kendall estimating equation, MS. Thesis, Georgia State University, 2009.

[20] Miller, R. G., A trustworthy jackknife, Ann. Math. Stat., Vol. 35, pp. 1594-1605, 1964

[21] Owen, A. B., Empirical likelihood for linear models. The Annals of Statistics, Vol. 19, pp. 17251747, 1991.

[22] Owen, A. B., Empirical likelihood ratio confidence intervals for a single functional, Biometrika, Vol. 75, pp. 237-249, 1988.

[23] Owen, A. B., Empirical likelihood ratio confidence regions. The Annals of Statistics, Vol. 18, pp. 90$120,1990$.

[24] Parzen, M. I., Wei, L.J. and Ying, Z., A resampling method based on pivotal estimating functions, Biometrika, Vol. 81, pp. 341-350, 1994.

[25] Qin.,G and Jing,B.Y, Empirical likelihood for censored linear regression, Scand. J. Statist., Vol. 28, pp. 661-673, 2001.

[26] Qin, J. and Lawless, J., Empirical likelihood and general estimating equations, The Annals of Statistics, Vol. 22, pp. 300-325, 1994.

[27] Quenouille, M.H. , Note on bias in estimation, Biometrika, Vol. 43, pp. 353-360, 1956.

[28] Ren, J., Weighted empirical likelihood in some two-sample semiparametric models with various types of censored data, Annals of Statistics, Vol. 36, pp. 147-166, 2008.

[29] Shao, J. and Tu, D., The Jackknife and Bootstrap, Springer-Verlag, 1995. 
[30] Shi, X, The approximate independence of jackknife pseudo-values and bootstrap methods, Journal of Wuhan Institute Hydra-Electric Engineering, Vol.2, pp. 83-90, 1984.

[31] Subramanian, S., Censored median regression and profile empirical likelihood, Statistical Methodology, Vol. 4, pp. 493-503, 2007.

[32] Thomas, D. R. and Grunkemeier, G. L.,Confidence interval estimation of survival probabilities for censored data, J. Amer. Statist. Assoc. Vol. 70, pp. 865-871, 1975.

[33] Tsiatis,A.A., Estimating regression parameters using linear rank tests for censored data, Ann. Statist, Vol. 18, pp. 354-372, 1990.

[34] Tukey, J.W , Bias and confidence in not-quite large samples, Annals of Mathematical Statistics, Vol. 29, pp. 614, 1958.

[35] Wei et al., Linear regression analysis of censored survival data based on rank tests, Biometrika, Vol. 77, pp. 845-851, 1990.

[36] Wood, A.T.A., Do, K.A. and Broom, N.M., Sequential linearization of empirical likelihood constraints with application to U-statistics, J. Comput. Graph. Stat.,Vol. 5, pp. 365-385, 1996.

[37] Ying, Z., A large sample study of rank estimation for censored regression data, Ann. Statist., Vol. 21, pp. 76-99, 1993.

[38] Zhao, Y., Empirical likelihood inference for the accelerated failure time model, Statistics and Probability Letters, Vol. 81, issue 5, pp. 603-610, 2011.

[39] Zhao, Y., and Chen, F., Empirical likelihood inference for median regression model via nonparametric kernel estimation, Journal of Multivariate Analysis, Vol.99, pp.215-231, 2008. 
[40] Zhao,Y., and Huang, Y., Test-based interval estimation under the accelerated failure time model, Communications in Statistics-Simulation and Computation, Vol.36, pp.593-605, 2007.

[41] Zhao,Y., and Yang S., Testing treatment effect via empirical likelihood using weighted log rank tests, Statistics and Probability Letters, Vol.77, pp.1385-1393, 2007.

[42] Zhou.M., Empirical likelihood analysis of the rank estimator for the censored accelerated failure time model, Biometrika, Vol. 92, pp.492-498, 2008.

[43] Zhou,M. and Li,G., Empirical likelihood analysis of the Buckley-James estimator, Journal of Multivariate Analysis, Vol. 99, pp. 649-664, 2008. 


\section{APPENDIX: MATLAB CODES}

\section{Matlab code:}

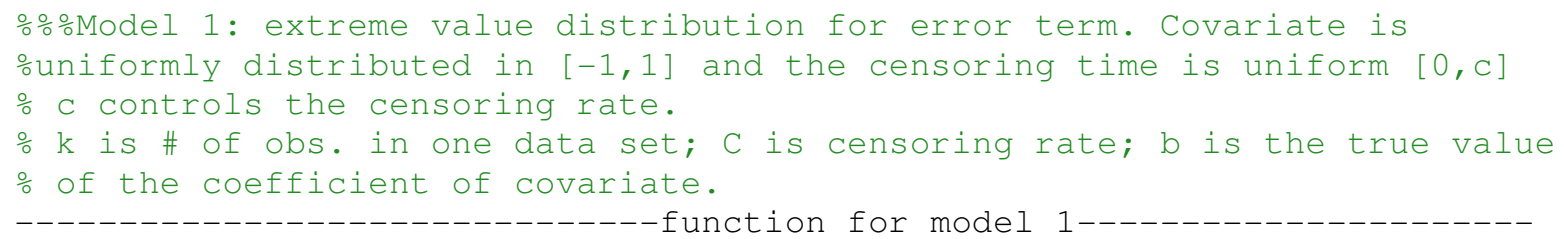




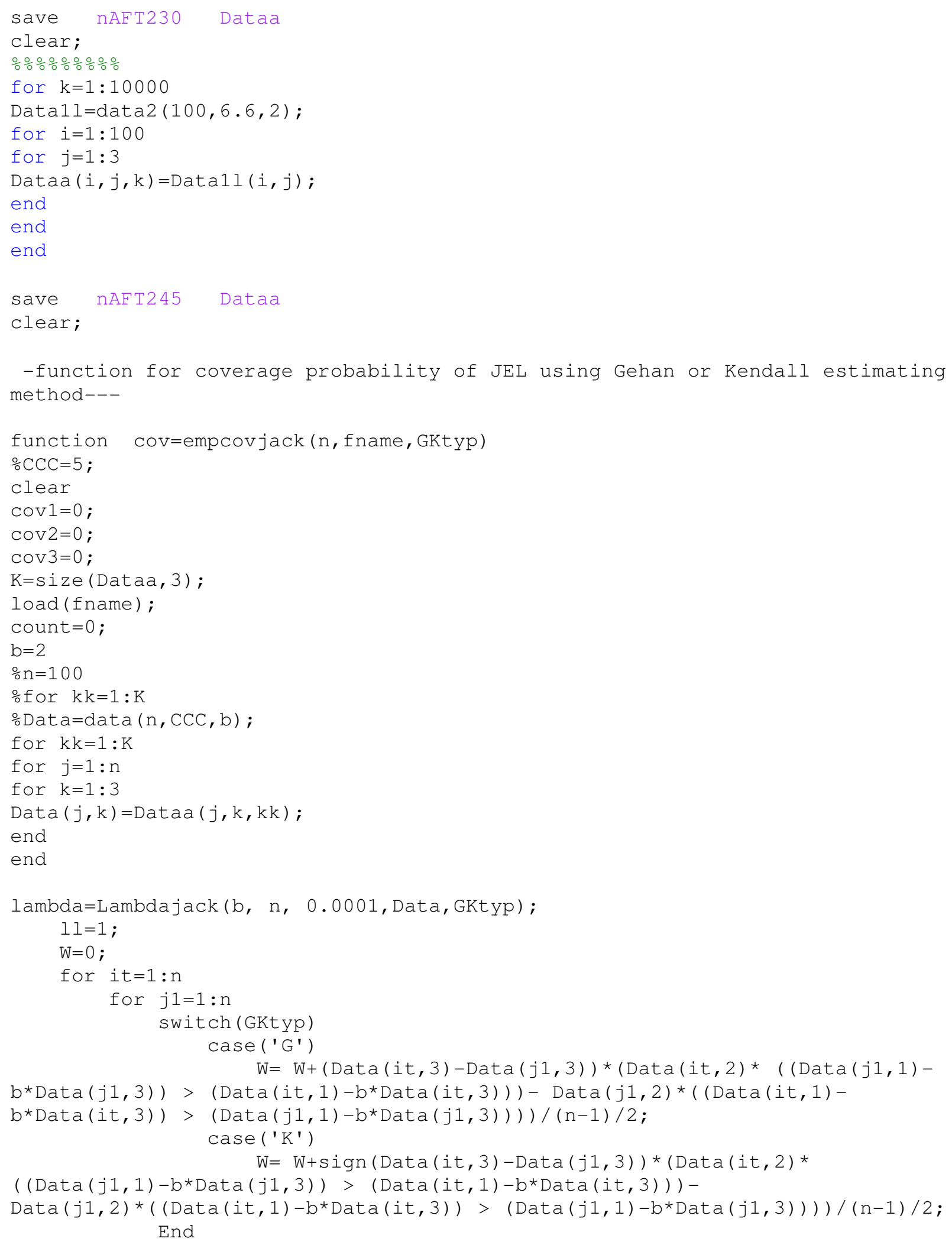


end

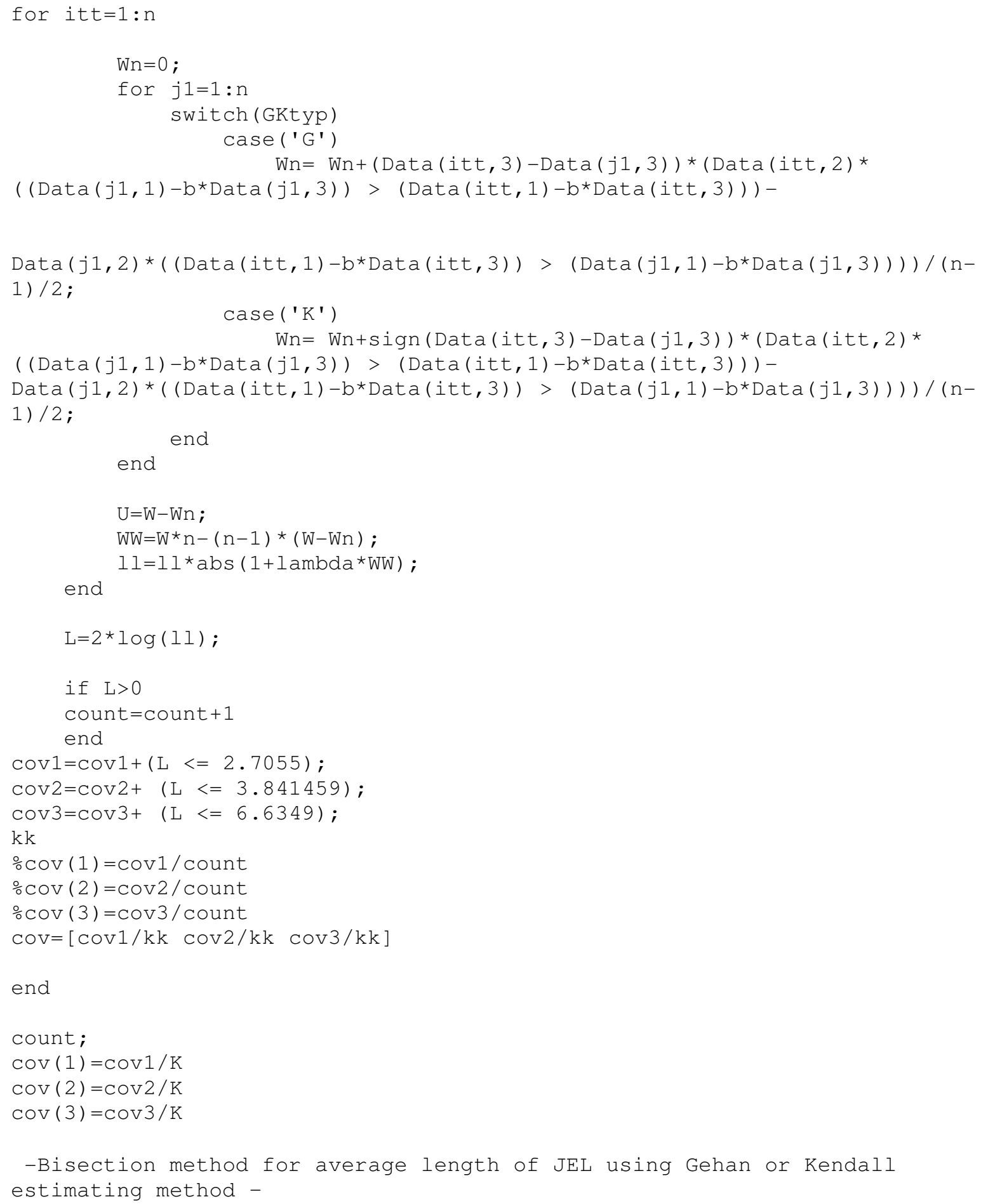




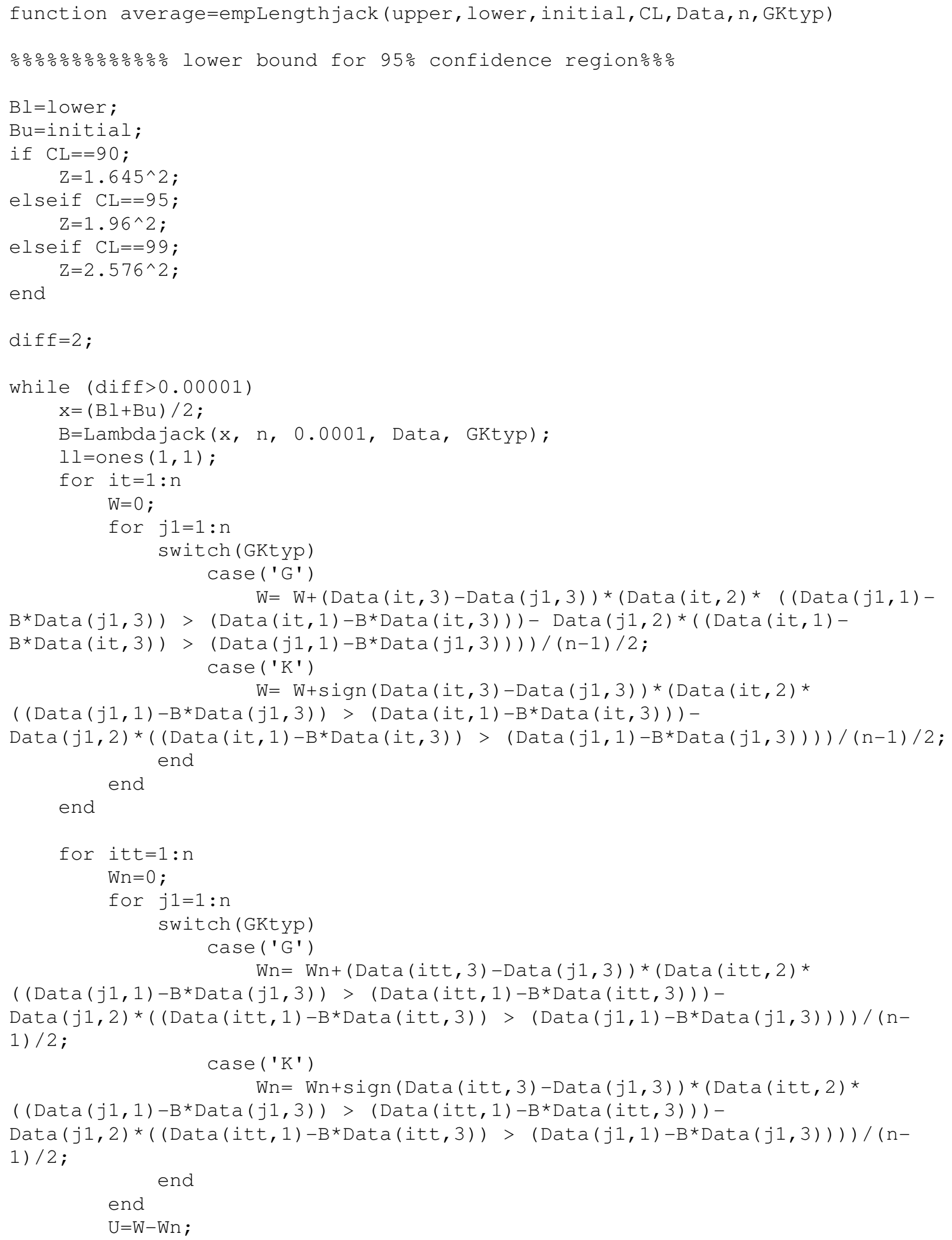


$\mathrm{WW}=\mathrm{W}^{*} \mathrm{n}-(\mathrm{n}-1)$ * $(\mathrm{W}-\mathrm{Wn})$;

$11=11 * \operatorname{abs}(1+B * W W)$;

end

$\mathrm{L}=2 * \log (11)$;

$$
\begin{aligned}
& \text { if } \begin{array}{l}
\quad<Z \\
B u=x ;
\end{array} \\
& \text { else } B l=x ; \\
& \text { end } \\
& \text { diff=Bu-Bl; }
\end{aligned}
$$

end

bhat $\mathrm{I}=\mathrm{Bu}$;

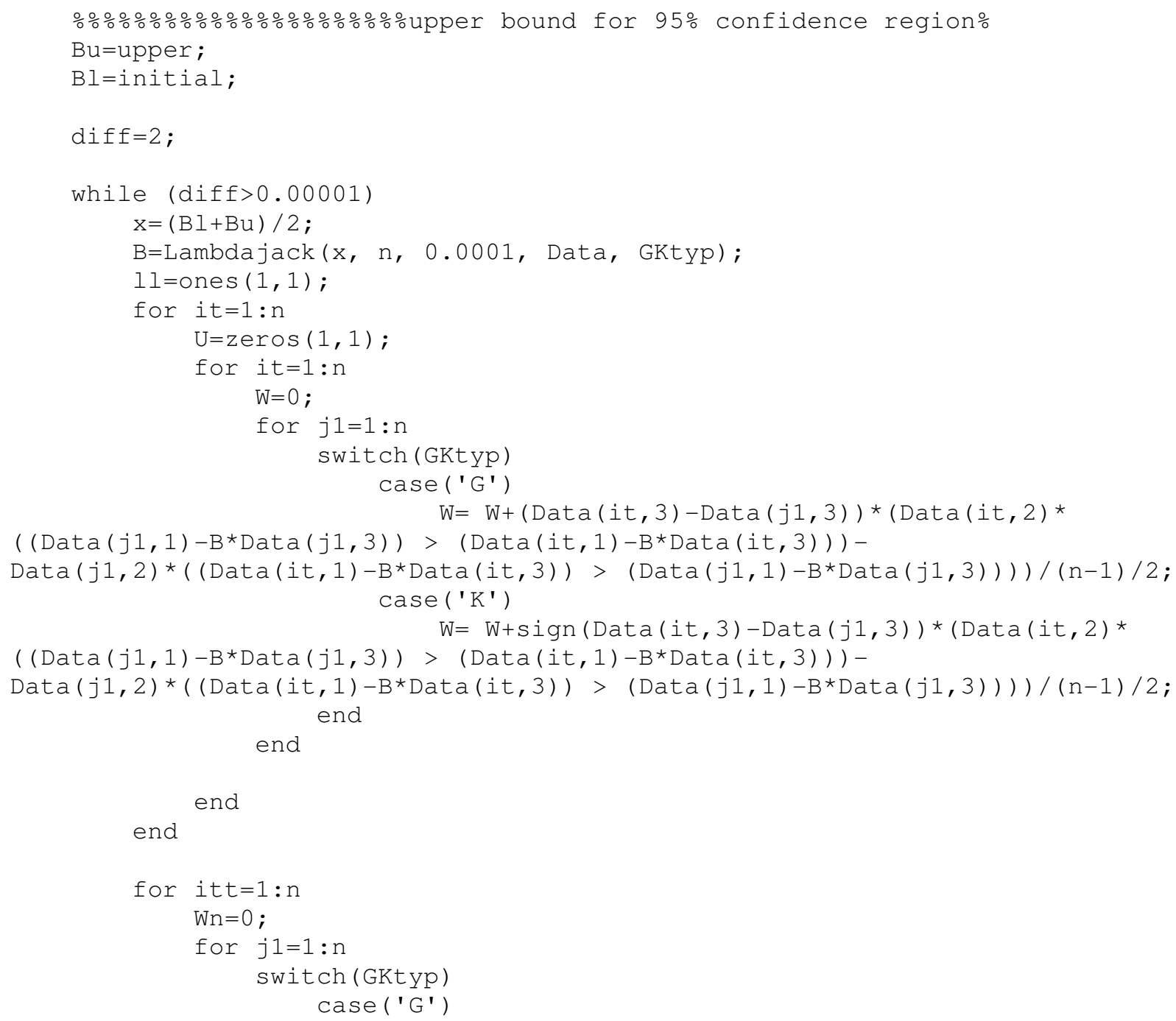


$((\operatorname{Data}(j 1,1)-B * \operatorname{Data}(j 1,3))>(\operatorname{Data}($ itt, 1$)-B * \operatorname{Data}(i t t, 3)))-$

$\operatorname{Data}(j 1,2) *((\operatorname{Data}(i t t, 1)-B * \operatorname{Data}(i t t, 3))>(\operatorname{Data}(j 1,1)-B * \operatorname{Data}(j 1,3)))) /(n-$ 1) $/ 2$;

$$
\text { case ( ' } \mathrm{K} \text { ') }
$$

$W n=W n+\operatorname{sign}(\operatorname{Data}($ itt, 3$)-\operatorname{Data}(j 1,3)) *(\operatorname{Data}($ itt, 2$)$ *

$((\operatorname{Data}(j 1,1)-B * \operatorname{Data}(j 1,3))>(\operatorname{Data}(i t t, 1)-B * \operatorname{Data}(i t t, 3)))-$

$\operatorname{Data}(j 1,2) *((\operatorname{Data}(i t t, 1)-B \star \operatorname{Data}(i t t, 3))>(\operatorname{Data}(j 1,1)-B \star \operatorname{Data}(j 1,3)))) /(\mathrm{n}-$ 1) $/ 2$;

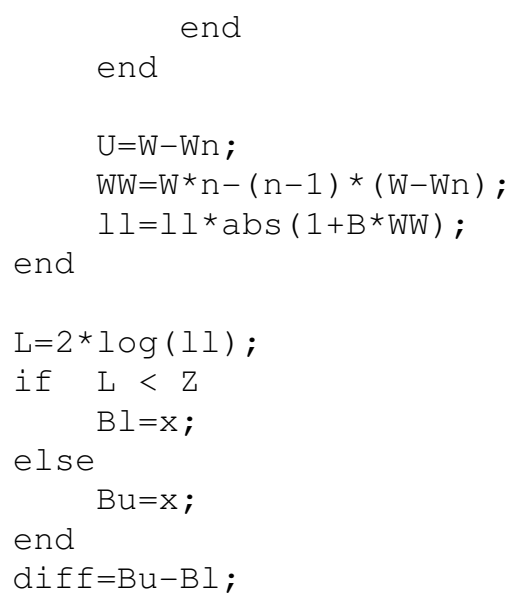


$((\operatorname{Data}(j 1,1)-\mathrm{b} * \operatorname{Data}(j 1,3))>(\operatorname{Data}(i t, 1)-\mathrm{b} * \operatorname{Data}(i t, 3)))-$

$\operatorname{Data}(j 1,2) *((\operatorname{Data}(i t, 1)-b * \operatorname{Data}(i t, 3))>(\operatorname{Data}(j 1,1)-\mathrm{b} * \operatorname{Data}(j 1,3)))) /(n-1) / 2 ;$ end

end

end

for $i t t=1: n$

$\mathrm{Wn}=0$;

for $j 1=1: n$

switch (GKtyp)

case ('G')

$W n=W n+(\operatorname{Data}(i t, 3)-\operatorname{Data}(j 1,3)) *(\operatorname{Data}($ itt, 2$)$ *

$((\operatorname{Data}(j 1,1)-\mathrm{b} * \operatorname{Data}(j 1,3))>(\operatorname{Data}($ itt, 1$)-\mathrm{b} * \operatorname{Data}($ itt, 3$)))-$

$\operatorname{Data}(j 1,2) *((\operatorname{Data}(i t t, 1)-b * \operatorname{Data}(i t t, 3))>(\operatorname{Data}(j 1,1)-\mathrm{b} * \operatorname{Data}(j 1,3)))) /(\mathrm{n}-$ $1) / 2$;

$$
\text { case (' } \mathrm{K} \text { ') }
$$

$W n=W n+\operatorname{sign}(\operatorname{Data}(i t t, 3)-\operatorname{Data}(j 1,3))$ (Data $($ itt, 2$)$ *

$((\operatorname{Data}(j 1,1)-\mathrm{b} * \operatorname{Data}(j 1,3))>(\operatorname{Data}(i t t, 1)-\mathrm{b} * \operatorname{Data}(i t t, 3)))-$

$\operatorname{Data}(j 1,2) *((\operatorname{Data}(i t t, 1)-\mathrm{b} * \operatorname{Data}(i t t, 3))>(\operatorname{Data}(j 1,1)-\mathrm{b} * \operatorname{Data}(j 1,3)))) /(\mathrm{n}-$

$1) / 2$;

end

end

$\mathrm{U}=\mathrm{W}-\mathrm{Wn}$;

$\mathrm{WW}=\mathrm{W} * \mathrm{n}-(\mathrm{n}-1)$ * $(\mathrm{W}-\mathrm{Wn})$;

$\mathrm{x}=\mathrm{x}-\left(\mathrm{WW} /\left(1+\mathrm{Bol} \mathrm{d}^{\star} W W\right)\right)^{\wedge} 2$;

end

ㅇor $i t=1: n$

$\div \mathrm{W}=0$;

\% for $j 1=1: n$

$\circ W($ it $)=W($ it $)+(\operatorname{Data}(i t, 3)-\operatorname{Data}(j 1,3)) *(\operatorname{Data}(i t, 2) *((\operatorname{Data}(j 1,1)-$

$\mathrm{b} * \operatorname{Data}(j 1,3))>(\operatorname{Data}(i t, 1)-\mathrm{b} * \operatorname{Data}(i t, 3)))-\operatorname{Data}(j 1,2) *((\operatorname{Data}(i t, 1)-$

$\mathrm{b} * \operatorname{Data}(\mathrm{it}, 3))>(\operatorname{Data}(j 1,1)-\mathrm{b} * \operatorname{Data}(j 1,3)))) /(\mathrm{n}-1) / 2$;

ond

$\frac{\circ}{o}=x-(W(i t) /(1+B o l d * W(i t)))^{\wedge} 2$;

응

-- function for Scoreltrans of JEL using Kendall or Gehan estimating method--

function S=Scoreltransjack (b,Bold, n, Data, GKtyp)

$\mathrm{S}=0$;

$\circ \mathrm{W}=\operatorname{zeros}(1, \mathrm{n})$;

$\mathrm{W}=0$;

for $i t=1: n$

for $j 1=1: n$ 


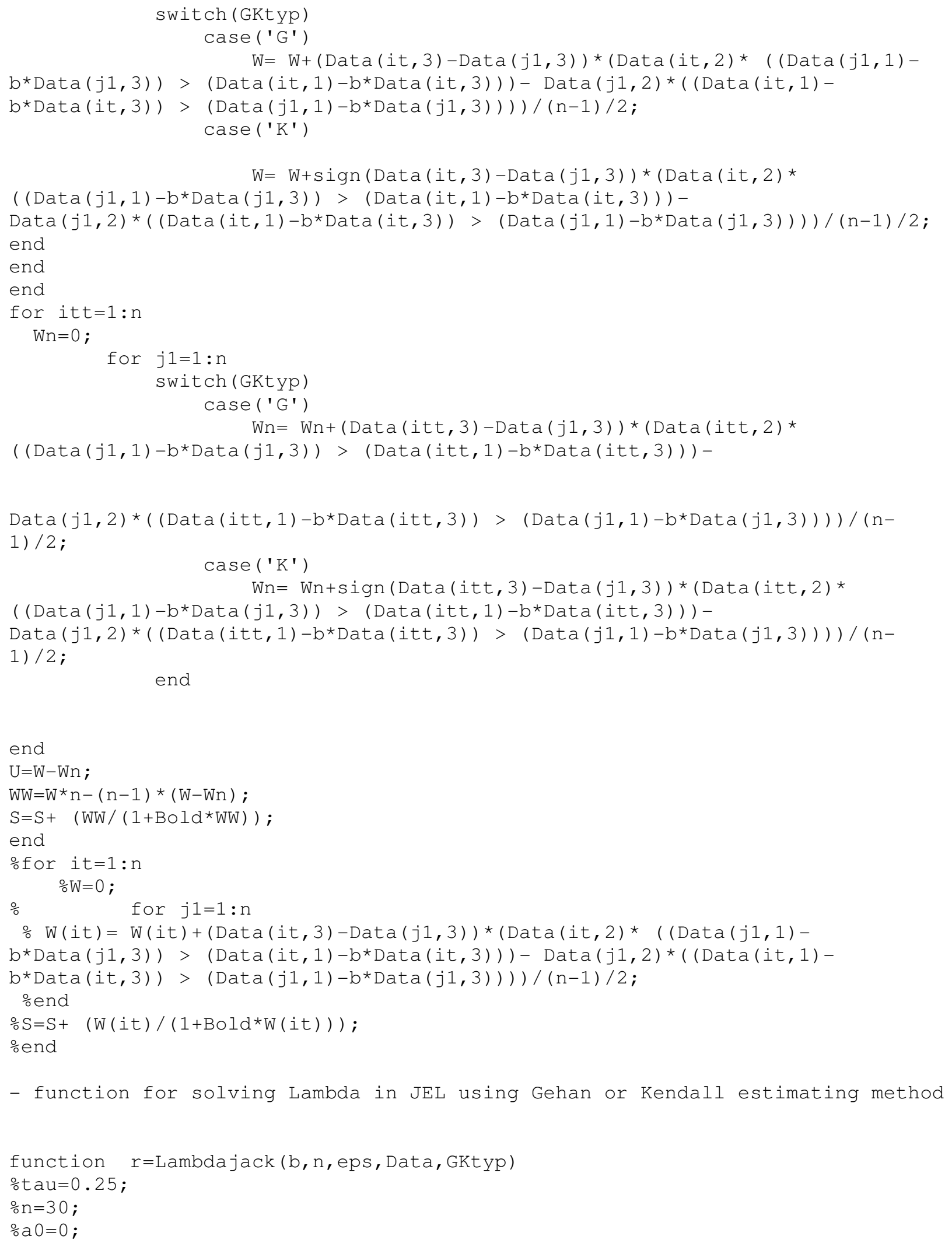


$\div a 1=-0.25$ ；

ㄴtau=0.25;

$\therefore \mathrm{CCC}=5$;

$\mathrm{Bold}=0$;

while (norm(Scoreltransjack (b, Bold, n, Data, GKtyp)) >eps)

Bold=Bold-inv (Jacobtransjack (b, n, Bold, Data, GKtyp)) * ...

count $=0$;

Scoreltransjack (b,Bold, n, Data, GKtyp) ;

end

$\mathrm{r}=\mathrm{Bold}$;

kkkk=norm (Scoreltransjack (b,Bold, n, Data, GKtyp) ); 